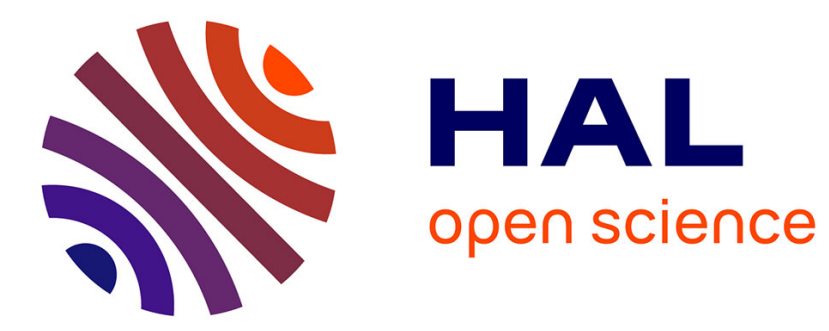

\title{
Présentation. Pour une épistémologie critique
}

\author{
Marie-Anne Paveau
}

\section{To cite this version:}

Marie-Anne Paveau. Présentation. Pour une épistémologie critique. Semen - Revue de sémiolinguistique des textes et discours, 2012, 34, pp.7-16. hal-00773189

\section{HAL Id: hal-00773189 \\ https://hal.science/hal-00773189}

Submitted on 11 Jan 2013

HAL is a multi-disciplinary open access archive for the deposit and dissemination of scientific research documents, whether they are published or not. The documents may come from teaching and research institutions in France or abroad, or from public or private research centers.
L'archive ouverte pluridisciplinaire HAL, est destinée au dépôt et à la diffusion de documents scientifiques de niveau recherche, publiés ou non, émanant des établissements d'enseignement et de recherche français ou étrangers, des laboratoires publics ou privés. 


\title{
Présentation \\ Pour une épistémologie critique
}

\author{
Marie-Anne Paveau
}

\section{«Voir ses yeux ». Une réflexivité créative}

Éric Didier est psychanalyste et reçoit surtout des enfants. Il a publié récemment un ensemble de conférences prononcées en Chine en 2010, sous le titre : Moi, je laisse faire, je regarde les étincelles. La première contient un paragraphe intitulé "Pourquoi je vois pas mes yeux? », où il associe les enfants, les chercheurs et les artistes dans leur remise en cause des évidences du monde. Chercher à "voir ses yeux», destituer l'ordinaire pour maintenir ouverte la question du sens, ce sont pour lui des occupations communes à ceux qui s'éveillent au monde et ceux qui essaient de le comprendre :

S'il y a un trait spécifique au moment de l'enfance, c'est la capacité de s'étonner. On peut parier que la première question qui pourrait être posée par un nouveau-né au moment où il arrive au monde serait « Mais qu'est-ce que c'est que ça ? »Cette question, il va la poser un peu plus tard, quand il sera enfant; il va la poser à la moindre occasion. À l'âge adulte, cette capacité de s'étonner va le plus souvent disparaitre, sauf pour les chercheurs et les artistes qui vont, eux, garder ce trait de l'enfance, cette capacité de questionner ce qu'ils rencontrent. Je crois que la très grande majorité des humains se bornent à prendre ce qui arrive comme une donnée incontestable, non questionnable, une donnée qu'il faut subir même si on doit s'en plaindre. Un bel exemple d'un questionnement radical d'un enfant, c'est celui d'un ami dont les parents m'ont raconté qu'il n'a pas parlé, qu'il n'a pas prononcé un mot pendant quatre ans. Un jour, à table, il a posé cette question devant ses parents, ce fut sa première parole : "Pourquoi je vois pas mes yeux? » Sacrée question! Les enfants sont d'abord des explorateurs qui vont questionner et subvertir les objets que les adultes ont depuis longtemps appris à voir sous l'angle de leur utilité, ou tout simplement, comme des « évidences ». Les enfants font pipi dans les pots de fleurs. J'ai vu une petite fille qui connectait un casque hi-fi à une bouteille de vin! Destitution de l'objet utile. Elle fait écouter de la musique à une bouteille de vin ou elle fait boire, elle enivre la musique, c'est-à-dire qu'avec deux objets très différents, elle fabrique un nouvel objet. Cela, nous, nous ne savons plus le faire (Didier 2011 : 18-19).

Cette conception repose sur des catégories sans doute un peu stéréotypées, mais la description du rapport au monde est intéressante et parlante pour les travaux rassemblés ici : connecter un casque hi-fi à une bouteille de vin, c'est un peu, en effet, l'objectif de ce numéro. Comme ils ne sont plus des enfants, les auteurs de cette livraison proposent plutôt de 
destituer l'ordinaire des mainstreams, de réinterroger les évidences, de faire en un mot un pas de côté qui permette de modifier la perspective afin de construire de nouveaux regards sur les questions posées par les linguistiques du texte, du discours et de l'interaction (désormais TDI). Cet écart n'est pas gratuit, mais veut répondre aux modifications épistémologiques repérables dans ces domaines ces dix ou même vingt dernières années, en particulier celles qui impliquent la réalité, le contexte, l'environnement, l'action. Ces réponses passent par une critique constructive des théories et méthodologies courantes, et le désir de présenter et de diffuser des approches moins connues, et par conséquent moins disciplinarisées et moins didactisées. C'est la perspective de l'épistémologie critique, que nous définissons comme une observation réflexive des théories et méthodes qui aboutissent à l'élaboration des connaissances. Le terme critique n'est pas à prendre au sens commun de contestation de résultats ou destruction d'arguments, mais bien au sens circulaire de retour des théories et des méthodes sur elles-mêmes (essayer de "voir ses yeux ") ou latéral d'effectuation d'un écart qui permet de modifier les perspectives. La critique est un outil de la réflexivité en même temps qu'elle en est un des fondements.

L'épistémologie, en particulier critique, est peu développée dans les disciplines TDI, et elle est principalement mobilisée par les réflexions sur les méthodes d'enquête et les postures des chercheurs en sociolinguistique (par exemple Robillard (dir.) 2009, Boyer (dir.) 2010). Il faut donc s'inspirer des questionnements d'autres disciplines : dans le champ de la philosophie de la connaissance, Ernest Sosa, l'une des figures importantes de la "virtue epistemology", propose une distinction très intéressante entre deux types de savoir, le "savoir animal" et le "savoir réflexif" :

One has animal knowledge about one's environment, one's past, and one's experience if one's judgements and beliefs about these are direct responses to their impact - e.g., through perception or memory - with little or no benefit or reflection or understanding. One has reflective knowledge if one's judgement or belief manifest not only such direct response to the fact known but also understanding of its place in a wider whole that includes one's belief and knowledge of it and how these come about (Sosa $1991: 240$ ).

Le savoir réflexif est évidemment mieux garanti que le savoir animal, la valeur du dernier n'étant cependant pas niée. Mais le premier est soutenu par une « vertu intellectuelle », notion développée par Aristote, oubliée ensuite au profit d'un idéal objectiviste, voire positiviste, mais réhabilitée dans certains développements de la philosophie de la connaissance à partir des années 1950 : "For reflective knowledge you need moreover an epistemic perspective that licenses your belief by its source in some virtue or faculty of your own " (Sosa 1991: 277). Le savoir réflexif est donc celui qui bénéficie d'une perspective, d'un regard de l'agent, le "knower", qui examine son savoir et le teste, en quelque sorte, à partir de ses facultés propres. Les critiques de l'état actuel des sciences humaines et sociales en France et ailleurs sont nombreuses, mettant en exergue en particulier l'extrême parcellisation des savoirs et la spécialisation des chercheurs. C'est le constat que fait Bernard Lahire, regrettant dans Monde pluriel que "l'enfermement disciplinaire», la trop grande division du travail en sciences humaines et l'hyperprofessionnalisation empêchent les chercheurs de prendre en compte les « liens d'interdépendance » entre leurs objets, les «couplages » et les circulations entre les différents domaines des pratiques sociales :

Tous les grands chercheurs inventifs [...] ont su allier rigueur de la pensée et ouverture maximale d'esprit vis-à-vis des disciplines connexes, avec le souci de ne jamais empêcher la mise en œuvre d'un ars inveniendi par l'application aveugle de démarches ou de méthodes disciplinaires entièrement routinisées. C'est parce qu'ils étaient davantage préoccupés par la construction scientifique pertinente de leur objet [...] que par le respect des frontières disciplinaires, qu'ils ont pu faire progresser, chacun à leur façon, les sciences humaines et sociales (Lahire 2012 : 329-330). 
C'est aussi la position d'Alexandre Dorna, dressant un tableau critique de l'état des SHS actuellement, à partir d'un examen de la psychologie : selon lui, la parcellisation des savoirs, la promotion des microthéories, le recours trop systématique au quantitativisme et un investissement trop important dans une vérité de la technologie entrainent « l'épuisement des grands paradigmes unitaires d'une part, et la fragmentation en disciplines autosuffisantes » (Dorna 2008 : en ligne) ${ }^{1}$. En analyse du discours par exemple, le travail sur certains objets constitue le mainstream de la discipline et assurent par là l'intégration et la reconnaissance des chercheurs dans le domaine ${ }^{2}$. Rares sont les interrogations sur le phénomène : pourquoi cette concentration? cette légitimité ? cette nécessité, presque, de travailler sur certaines question pour que son travail soit reconnu comme étant de l'analyse du discours $?^{3}$ Et rares en sont les critiques bien sûr, dont l'absence définit justement la notion même de mainstream ${ }^{4}$. Ce phénomène correspond bien à ce que Jay Rothman et ses collaborateurs appellent avec d'autres la densité des disciplines: "As disciplines grow and become dense with theorists, there is an overcrowding in the academic field with many scholars studying the same patrimony and asking the same questions. Such density is not characterized by innovation » (Rothman et al. 2001 : 65). Cette densité finit par déboucher sur l'épuisement des paradigmes dont parle Alexandre Dorna, mais également celui des objets eux-mêmes. Les connaissances n'avancent pas dans l'accord consensuel et le développement du mainstream; elle se renouvellent dans ces "pas de côté » souvent scientifiquement impertinents qui permettent d'inventer de nouveaux chemins et cheminements. Les auteurs rappellent alors la richesse d'un concept élaboré par Mattei Dogan et Robert Pahre (1990), qui ressemble bien à la description empirique d'Éric Didier : la « marginalité créative (creative marginality) » :

As some scholars move away from the mainstream and toward the margins of the field, they begin to look toward the margins of other fields that may overlap and fill in those gaps. This interaction outside of disciplinary boundaries provides the grounds for intellectual crossfertilization, and it is often the site at which innovation occurs. "Not only are the margins less densely populated, providing more room to grow, but successful combinations of material from two sub fields typically allows greater scope for creativity. In fact, the greatest accumulation of incremental advances takes place at the intersection of fields $»^{5}$ (Rothman et al. $2001: 65$ ).

Dans ce numéro, les approches présentées ne sont pas forcément « marginales » au sens fort du terme, mais elles reposent toutes sur une conviction: la nécessité de (re)penser nos disciplines par rapport à des réalités évolutives, réalités tant des dispositifs théoriques et des postes d'observation des sciences humaines que du monde et des environnements désormais complexes qui le constituent.

\section{Postdualisme, poststructuralisme, postdisciplinarité}

Tous ces post- énumérés à la file veulent indiquer que cette « crise » de la densité des sciences humaines est peut-être le symptôme d'un passage à d'autres repères pour l'humanité : on

\footnotetext{
${ }^{1}$ La revue C@hiers de psychologie politique n'indique pas les paragraphes, d'où l'absence de pagination de cette référence.

2 Pour des détails et une réflexion sur le mainstream contemporain en analyse du discours, voir Paveau 2012.

${ }^{3}$ Dans un précédent numéro de Semen en 2010, j'ai raconté qu'une remarque d'un doctorant en ce sens était à l'origine de mon travail d'histoire et de description critiques de la notion de dialogisme : « Mais madame, moi, le dialogisme, sur mon corpus, ça ne marche pas, je n'y arrive pas » (Paveau 2010b)

4 Paveau 2010a.

5 Dogan, Pahre 1990.
} 
mentionne souvent la révolution numérique actuelle, mais on ne mesure sans doute pas encore les modifications concrètes qu'elle implique, en particulier dans notre rapport à l'écriture et plus généralement aux discursivités, qui commencent à effacer la frontière entre production humaine et production artefactuelle; la robotisation de l'humain reste encore pour beaucoup d'entre nous de l'ordre de la science-fiction ou du mythe mais des réalisations bien concrètes montrent pourtant que nous sommes déjà entrés dans l'ère de l'humanité augmentée; d'une manière générale, "l'exception humaine », selon l'expression de JeanMarie Schaeffer (2007), s'affaiblit, et avec elle l'exception langagière qui en est le corollaire immédiat. Toute modification de l'humain ou de son statut entraine une modification de sa capacité primordiale, le langage, et, pour cette raison, les linguistes devraient peut-être se montrer particulièrement attentifs aux paradigmes en post-.

Dans ce numéro, les auteurs ont essayé de soutenir cette vigilance en appuyant leurs analyses sur la question des binarismes traditionnels, celle des (re)configurations inter- , pluri-, transet postdisciplinaire des disciplines et celle des acquis des sciences cognitives, en particulier dans leur version hétérodoxe sociale et culturelle.

\section{Au-delà des binarismes traditionnels}

On doit se demander comment est pris en compte dans les disciplines des SHS et en particulier les sciences du langage et leurs disciplines contributives, l'effacement des grands binarismes corps/esprit, langue/monde, humain/technique, texte/contexte, linguistique/extralinguistique. Ces remises en cause impliquent un certain refus du logocentrisme (défini comme l'ensemble des approches linguistiques qui se font à partir des productions verbales) et de l'égocéphalocentrisme (terme proposé par le sociologue JeanClaude Kaufmann et diffusé par Christian Brassac (2007), désignant une approche à partir de l'énonciateur et de son esprit) et nécessitent de considérer des productions verbales comme ouvertes sur les réalités de l'action, du travail, de la technique et de la technologie, de la culture, des environnements, des perceptions, etc. Alors que le débat sur l'extended mind est riche et constamment alimenté en philosophie, depuis notamment l'article fondateur de Andy Clark et David Chalmers (1998), il n'existe pas, en linguistique, de discussion sur ce qui pourrait être un «extended logos » ou «extended language» : la nature de l'objet langue, fixé depuis Saussure, n'est pas mis en débat, et pourtant, actuellement plus que jamais se pose la question du métissage technique du logos : la langue n'est-elle vraiment « que » de la langue ? Les auteurs de ce numéro apportent des réponses variées à cette question, en défendant l'idée d'une approche des productions verbales ouverte sur l'ensemble de l'environnement et non réduite au seul fil de la phrase ou du discours. Cette perspective pose rapidement la question disciplinaire, puisqu'une approche globale des productions verbales en contexte modifie les contours des disciplines stricto sensu.

\section{Une interdisciplinarité a-disciplinaire?}

Dans sa contribution au collectif dirigé par Frédéric Darbellay et Theres Paulsen sur l'interdisciplinarité (2008), Jacques Lévy insiste sur les risques de clôture des disciplines, ne leur permettant pas une véritable interdisciplinarité :

[...] la tendance pour chaque discipline à se créer une cosmogonie totalisante, ignorant celle des autres et prétendant pratiquer l'«interdisciplinarité disciplinaire " tout en restant farouchement accroché à son pré carré, est massivement présente dans les sciences sociales. Ce splendide isolement a pour effet de rendre très difficile la rencontre entre des territoires du savoir bornés par des frontières étanches et, pire encore, opérant sur des plans déconnectés les uns des autres. La définition que chaque discipline donne de son domaine est le plus souvent incompatible avec l'existence des autres. Cette absence de symétrie dans les 
épistémologies disciplinaires montre que, à cet égard, les sciences sociales (mais probablement pas elles seulement) en sont à un «stade infantile » de leur histoire (Lévy 2008 : 198-199).

Pour lui, l'avenir des disciplines est la postdisciplinarité, reposant sur une sorte de "socle commun ». Comme Bernard Lahire ou Alexandre Dorna, ou encore Frédéric Darbellay, il estime que le travail le plus fécond en sciences humaines et sociales passe par des objectifs, les compétences et les savoirs partagés préalablement :

Il serait donc temps de passer, pour le «continent des sciences s'intéressant aux humains en tant qu'ils vivent ensemble (le champ des sciences humaines et sociales ) », de la fragmentation à une «maison commune » ou à un « espace public» qui valoriserait les ressemblances et les convergences. Il s'agirait d'inverser l'attitude actuelle (on construit la plus grande part des savoirs séparément puis on regarde s'il peut y avoir des points communs) et penser l'horizon de la recherche sous la figure de la post-disciplinarité, c'est-à-dire d'une a-disciplinarité principielle au sein de cet ensemble (Lévy 2008 : 199-200).

Les articles de ce numéro ne font pas forcément référence à l'interdisciplinarité sous ce vocabulaire de l'a-disciplinarité ou de la postdisciplinarité. Néanmoins, ils présentent tous des entrées qui font une place véritable à l'articulation de différentes disciplines. Par " articulation véritable », on veut dire : qui permettent qu'une discipline soit modifiée par une autre, et pas seulement « colorée » par elle, qui font réellement travailler les disciplines en cocontribution et non en juxtaposition. On trouvera par exemple une articulation serrée de la linguistique argumentative, de la rhétorique, des sciences cognitives et de l'anthropologie chez Emmanuelle Danblon, des croisements structurels entre communication, psychologie, éthologie, sciences de l'éducation, anthropologie, chez Ingrid de Saint-Georges, une coopération entre épistémologie, histoire des sciences et rhétorique chez Maria Załęska ; un tressage entre linguistique textuelle, sémantique cognitive et philosophie chez Guy AchardBayle, entre théorie du discours, philosophie, sciences du numérique et cognition sociale chez Marie-Anne Paveau, sciences de la communication, humanités numériques et sciences de l'éducation chez Olivier Le Deuff, anthropologie, sciences de la communication, sciences de l'éducation, sociologie et didactique des langues chez Fred Dervin et Céline Tournebise.

\section{Les acquis des approches sociales de la cognition}

La majeure partie des contributions prend en compte, de manière explicite ou implicite, les acquis des sciences cognitives, ou de la cognition au sens plus général de construction des connaissances, dans les différentes disciplines concernées. Le paramètre cognitif a en effet produit certains changements de braquet intéressants qui permettent désormais de dépasser certaines options théoriques et méthodologiques, comme les approches structuralistes ou structuralisantes par exemple, ou une conception de l'interaction encore trop souvent réduite aux interactions des seuls protagonistes de l'énonciation. Le domaine de la cognition sociale en particulier, dans sa version distribuée, propose des dispositifs théoriques et méthodologiques pour penser à nouveaux frais la contribution des realia (réalités corporelles, objectales, environnementales) et des techniques (artefacts, outils informatiques, supports techniques de communication) à la production verbale (textes, discours, interactions). La source du regard se déplace, et, plutôt que de partir des productions verbales, qu'elles soient, textes, discours, ou interactions, les chercheurs rassemblés ici souhaitent prendre en compte le dispositif plus vaste dans lequel elles sont élaborées, qu'il soit appelé "réalité », " environnement», " contexte», «culture» ou «action». De même que, depuis les travaux d'Edwin Hutchins, c'est le cockpit tout entier qui se souvient de ses vitesses et non les seuls pilote et copilote (Hutchins 1994 [1991]), c'est ici la réalité sociale prise dans sa structure d'ensemble qui produit les énoncés, et non les seuls énonciateurs. 


\section{Cheminements dans les disciplines TDI}

Le numéro est organisé en deux parties. La première, "Disciplines et paradigmes », rassemble des points de vue qui font une place importante à l'historicité des disciplines et aux évolutions des regards théoriques sur les objets privilégiés des linguistiques TDI. Le lecteur suit d'abord avec Emmanuelle Danblon, dans "La rhétorique ou l'art de pratiquer l'humanité », les cheminements du modèle rhétorique d'Aristote, aux deux bouts antérieur et postérieur, de notre modernité argumentative. Comme en philosophie, les propositions d'Aristote constituent des réouvertures, voire des réinventions dans le champ des textes et des discours. Cette histoire non linéaire des théories, c'est l'objet qu'examine Maria Załęska dans “Ordre et chaos dans les disciplines", qui prend justement l'exemple de la rhétorique. Comment s'agencent et s'organisent les disciplines ? Comment évoluent-elles ? Et quels effets ont sur elles les contributions interdisciplinaires? Si l'ordre règne dans une discipline conçue comme un champ homogène et fermé, c'est le chaos, au sens positif du terme, qui définit l'ouverture et l'hétérogénéité de la postdisciplinarité. Le paradigme de la Mediated Discourse Analysis, examiné ensuite par Ingrid de Saint-Georges dans « Nouvelles épistémologies en analyse du discours et des interactions », pourrait constituer un bon exemple de postdiscipline. En effet, on y voit à l'œuvre un corps théorique et méthodologique qui n'est pas ou plus celui des différentes disciplines qui contribuent à ce champ, mais qui est propre à leur rencontre " chaotique ». La créativité est là, dans l'invention d'une approche qui dépasse les applications et les emprunts simples, pour construire une recherche métissée.

La seconde partie, "Réalités, techniques, cultures », se tourne vers les realia qui constituent les terrains et les objets des disciplines TDI. Dans «Vérité-Réalité-Naturalité : La Relation et l'Ancrage », Guy Achard-Bayle s'interroge sur la place que tiennent les trois notions de vérité, réalité et naturalité dans les sciences humaines et sociales, et par conséquent dans les sciences du langage, entendues au sens intégrateur de disciplines du logos. Le naturalisme est actuellement l'un des courants majeurs de l'ensemble des sciences, tant exactes qu'humaines, et les disciplines TDI doivent interroger leur fondations par rapport aux données naturalistes fournies essentiellement par les sciences cognitives. Ces fondations en sont modifiées, comme le montre Marie-Anne Paveau dans «Réalité et discursivité. D’autres dimensions pour la théorie du discours » : nous sommes entrés dans une ère postdualiste, qui considère la réalité comme un assemblage de données hétérogènes plus que comme une organisation de catégories discrètes. Cette perspective est mobilisable dans une théorie linguistique du discours afin de modifier et d'enrichir les notions de sujet, de contexte et de langue. Parmi ces données hétérogènes assemblées figure la technique, bien sûr, au cœur de la révolution numérique. Dans « Humanisme numérique et littératies », Olivier Le Deuff montre à quel point les humanités numériques en plein développement modifient le travail même de la recherche. Les données qui constituaient la littératie ont changé, et c'est vers une translittératie que nous nous dirigeons. Réalité, naturalité, technique, mais aussi culture : dans «Les rendez-vous ratés de l'interculturel en éducation? Une étude de cas de l'éducation à la communication interculturelle en Finlande ", Fred Dervin et Céline Tournebise se livrent à un examen critique de la notion d'interculturel, montrant comment cette notion est un construit, lui-même culturel. Les représentations que les acteurs ont de l'interaction avec des sujets d'autres cultures sont en décalage avec les recherches récentes sur la question : dans le domaine des interactions se joue aussi une évolution des conceptions, de la "solidité » des cultures nationales et locales, à la « liquidité » des circulations et des transferts.

Ce numéro est un ensemble de propositions. Il ne présente pas de nouvelles doctrines, encore moins de nouvelles organisations disciplinaires. Il ne prescrit rien. Il présente des pensées de 
l'hétérogène, de la fluidité, voire du chaos. Il se fraye un chemin de traverse un peu à l'écart des grandes routes des disciplines denses. Un petit sentier latéral, que ceux qui se sentent comme les enfants-chercheurs décrits par Éric Didier sont invités à emprunter.

\section{Références}

- Boyer H. (dir.), 2010, Pour une épistémologie de la sociolinguistique, Limoges, Lambert-Lucas.

- Brassac C., 2007, «Co-responsabilité cognitive et dissolution de frontières", In P. Hert, M. PaulCavallier (éds), Sciences et frontières. Délimitations du savoir, objets et passages. Fernelmont (BE) : Éditions Modulaires Européennes \& InterCommunications, 159-176.

- Clark A., Chalmers D., 1998, « The extended mind », Analysis 58 (1), 10-23.

- Darbellay F., Paulsen T., 2008, Le défi de l'inter- et transdisciplinarité : concepts, méthodes et pratiques innovantes dans l'enseignement et la recherche, Lausanne, Presses polytechniques et universitaires romandes.

- Didier É., 2011, Moi, je laisse faire, je regarde les étincelles. Cinq conférences sur la psychanalyse d'enfants, Paris, Petite CAPITALE.

- Dogan, M., Pahre R., 1990, Creative Marginality: Innovation at the Intersections of Social Sciences, Boulder, Westview Press.

- Dorna A., 2008, «Malaises et critiques en psychologie et en sciences sociales», Les cahiers psychologie politique 13, http://lodel.irevues.inist.fr/cahierspsychologiepolitique/index.php?id=117

- Hutchins E., 1994 [1991], "Comment le cockpit se souvient de ses vitesses » (trad. de «How a Cockpit Remembers its Speed »), Sociologie du travail 4, 461-473.

- Lahire B., 2012, Monde pluriel. Penser l'unité des sciences sociales, Paris, Seuil.

- Lévy J., 2008, « Sortir du pavillon disciplinaire, dans Darbellay F., Paulsen T., 2008, Le défi de l'interet transdisciplinarité : concepts, méthodes et pratiques innovantes dans l'enseignement et la recherche, Lausanne, Presses polytechniques et universitaires romandes, 194-217.

- Paveau M.-A., 2010a (dir.) : La théorie du discours. Fragments d'histoire et de critique, Semen 29, Besançon, PUFC, http://semen.revues.org/8756

- Paveau M.-A., 2010b : "La norme dialogique. Propositions critiques en philosophie du discours », Semen 29, p. 127-146, http://semen.revues.org/8793

- Paveau M.-A., 2012, "Que veut dire travailler en analyse du discours en France en 2011 ? Épistémologies, objets, méthodes », actes du colloque III Encontro Internacional de Estudos da Linguagem, $\quad$ septembre Pouso Alegre, http://www.cienciasdalinguagem.net/enelin/anais/texts/87.pdf

- Robillard D. (de), 2009 (coord.), Cahiers de sociolinguistique 14, "Réflexivité, herméneutique, vers un Paradigme de recherche ?" Rennes, PUR.

- Rothman J., Rothman R., Schwoebel M., 2001, «Creative marginality: Exploring the links between conflict resolution and social work », Peace and Conflict Studies 8-1 : 63-82.

- Schaeffer J.-M., 2007, La fin de l'exception humaine, Paris, Gallimard.

- Sosa E., 1991, Knowledge in Perspective: Selected Essays in Epistemology, Cambridge and NY: Cambridge University Press. 